Pacific Journal of Mathematics

FIELDS OF TOPOLOGICAL SPACE 


\title{
FIELDS OF TOPOLOGICAL SPACES
}

\author{
JOHN MACK
}

In their memoir "Representation of rings by sections", Memoirs, Amer. Math. Soc., Dauns and Hofmann introduce the concept of "field of uniform spaces" which provides an extremely useful setting in which a wide class of topological rings can be represented as rings of continuous sections. The Dauns-Hofmann theory uses a mixture of uniform and topological techniques to achieve its ends. The purpose of this note is to show that much of the Dauns-Hofmann theory can be developed using solely topological techniques without resort to the concept of field uniformity which is central to the Dauns-Hofmann approach. The theory developed here represents a natural extension of that of fibre bundles.

1. Definition. A field of sets is a quadruple $(E, B, Y, \Phi)$ where $E, B$, and $Y$ are sets and $\Phi$ is a family of maps $\phi: \operatorname{dom} \phi \rightarrow E$ from a subset of $B \times Y$ to $E$ (here dom $\phi$ denotes the domain of $\phi$ ) such that the following conditions are satisfied:

(A) $E=\bigcup_{\phi \in \Phi}$ image $\phi$

(B) $B=\bigcup_{\phi \in \Phi} \mathrm{pr}_{B}[$ dom $\phi]$ where $\mathrm{pr}_{B}$ denotes the projection of $B \times Y$ onto $B$.

(C) For $b_{r} \in B, y_{r} \in y$, and $\phi_{r} \in \Phi$ with $r=1,2$, the equality $\phi_{1}\left(b_{1}\right.$, $\left.y_{1}\right)=\phi_{2}\left(b_{2}, y_{2}\right)$ implies $b_{1}=b_{2}$.

A field of topological spaces is a field of sets $(E, B, Y, \Phi)$ in which $E, B$, and $Y$ are topological spaces and $\Phi$ is a set of continuous, open maps with open domain in $B \times Y$. The space $E$ will be called the field space and $B$, the base space. The maps $\phi$ are called co-ordinate maps.

REMARK. If $(E, B, Y, \Phi)$ is a field of topological spaces for which each $\phi \in \Phi$ is injective (and hence a homeomorphism of its domain onto its image), then the field is a fibre bundle in the sense of Steenrod [7]. Also, if $Y$ has the discrete topology then $(E, B, Y, \Phi)$ is a sheaf. Thus fibre bundles and sheaves are examples of fields of topological spaces. An example of a field of topological spaces that is neither a sheaf nor a fibre bundle is given below (Example D)

ExAmple A. For topological spaces $B$ and $Y$ let $E=B \times Y$ and $\phi$ be the identity map on $B \times Y$. Then $(E, B, Y, \phi)$ is a field of topological spaces.

Example B. Let $B$ be the circle $S^{1}, Y=[0,1]$ and $E$ be the Mobius band. Select distinct points $p_{1}, p_{2}$ from $B$ and let $\phi_{i}:\left[B \backslash\left\{p_{i}\right\}\right] \times$ 
$Y \rightarrow E$ be a homeomorphism onto an open subset of $E$ for $r=1,2$. Further assume $E$ is the union of the images of $\phi_{1}$ and $\phi_{2}$. Then ( $E$, $\left.B, Y,\left\{\phi_{1}, \phi_{2}\right\}\right)$ is a field of topological spaces.

Example C. Let $E$ be an $n$-manifold and $\Phi$ be an adequate set of coordinate maps from $E^{n}$ to $E$. If $B$ is a singleton and $\phi^{1}$ is defined by $\phi^{1}(b, y)=\phi(y)$, then $\left(E, B, E^{n}, \Phi^{1}\right)$ is a field of topological spaces where $\Phi^{1}=\left\{\phi^{1}: \phi \in \Phi\right\}$.

ExAmple D. Let $B$ be a compact Hausdorff space and $Y$ be the topological ring $C(B)$ of all continuous real valued functions on $B$, with the uniform norm. Set $E=B \times R$ ( $R$ is the real line) and define $\phi: B \times Y \rightarrow E$ by $\phi(b, f)=(b, f(b))$. Then $(E, B, Y, \phi)$ is a field of topological spaces.

Example E. Let $A$ be a topological ring, $B$ be an appropriate set of ideals (the set of primitive ideals for example) and $E=\mathbf{U}_{b \in B}\{b\} \times$ $A / b$. If $\phi: B \times A \rightarrow E$ is defined by $\phi(b, a)=(b, a+b)$, then $(E, B$, $A, \phi)$ is a field of sets. If $\hat{a}$ is defined by $\hat{a}(b)=(b, a+b)$ and the kernel of $B$ is $\{0\}$, then $a \rightarrow \hat{a}$ is a faithful representation of $A$. If $B$ and $E$ are topologized so that $\phi$ is a continuous, open map then each $\hat{a}$ is a continuous section. In that case $(E, B, A, \phi)$ becomes a field of topological spaces with respect to which the elements of $A$ are representable as continuous sections.

Examples $D$ and $E$ suggest the possibility of representing topological rings as rings of continuous sections over fields of topological spaces. It is this fact that motivates much of this paper. However, at the moment, we shall content ourselves with developing the topological aspects of fields of topological spaces and only in a later paper will we address ourselves to the representation problem for rings.

It is convenient in applying fields of topological spaces in special situations to allow $\Phi$ to have arbitrary cardinality. However, in developing the theory, it is also convenient to assume that $\Phi$ consists of a single element. The following theorem shows that there is no loss of generality in making the latter assumption.

2. THEOREM. If $(E, B, Y, \Phi)$ is a field of topological spaces then $(E, B, Y \times \Phi, \psi)$ is also such a field if $\Phi$ is assigned the discrete topology and $\psi$ is defined by $\psi(b, y, \phi)=\phi(b, y)$.

Proof. Note that dom $\psi=\bigcup_{\phi \in \Phi}(\operatorname{dom} \phi) \times\{\phi\}$ is open in $B \times Y \times$ $\Phi$. Also, it is clear that (A), (B), and (C) hold for $\psi$. Finally, it is a simple matter to check that $\psi$ is a continuous open map.

Notation convention. (i) We shall in the statement of theorems 
allow $\Phi$ to have arbitrary cardinality. However, whenever it is convenient to do so, in proofs we shall assume $\Phi$ to be a singleton.

(ii) If $G$ is a subset of $B \times Y$, then $\phi(G)$ shall mean the $\operatorname{set} \phi(G \cap$ dom $\phi)$. In particular $\operatorname{dom} \phi=\phi^{-1} \phi(B \times Y)$.

3. Lemma. If $(E, B, Y, \Phi)$ is a field of topological spaces then there exists a unique continuous open surjection $\pi: E \rightarrow B$ such that $\pi \circ \phi$ is the restriction to $\operatorname{dom} \phi$ of the projection of $B \times Y$ to $B$.

Proof. For $x \in E$, select $b \in B, y \in Y$ and $\phi \in \Phi$ so that $x=\phi(b, y)$. Such elements exist by axiom (A). Then define $\pi(x)=b$. Axiom (C) implies that $\pi$ is well defined and (B) shows that $\pi$ is surjective. Clearly $\pi \circ \phi(b, y)=b$ for all $(b, y) \in \operatorname{dom} \phi$; thus $\pi \circ \phi$ is the restriction to dom $\phi$ of the projection of $B \times Y$ to $B$. To prove that $\pi$ is continuous let $x \in E$ and $V$ be open in $B$ such that $\pi(x) \in V$. Select $\phi \epsilon$ $\Phi$ so that $x \in$ image $\phi$. Then $\phi[(V \times Y) \cap$ dom $\phi]$ is a neighborhood of $x$ whose image under $\pi$ is contained in $V$. Whence $\pi$ is continuous. That $\pi$ is an open map follows from the continuity of $\phi$ and the openness of $\pi \circ \phi$ for each $\phi \in \Phi$.

4. Definition. The map $\pi$ defined in the proof of Lemma 3 will be called the field projection. The set $\pi^{-1}(b)$ is the stalk over $b$ and a map $\sigma: B \rightarrow E$ will be called a section over the field $(E, B$, $Y, \Phi)$ if $\pi \circ \sigma$ is the identity map on $B$. A map $\sigma: U \rightarrow E$ from an open subset $U$ of $B$ to $E$ is a local section if $\pi \circ \sigma$ is the identity on $U$.

In their memoir [3] Dauns and Hofmann define a field of topological spaces to be a triple $(E, B, \pi)$ where $E$ and $B$ are space and $\pi: E \rightarrow B$ is a continuous open surjection such that

(I) $E=\bigcup$ image $\sigma: \sigma$ is a continuous local section with respect to $\pi$.

The term "field of topological spaces" as used in this note varies slightly from the Dauns-Hofmann meaning for this term. Our field of topological spaces represents a concept which lies between the Dauns-Hofmann field of topological spaces and their field of uniform spaces. The relations existing among these concepts are given by the next theorem and Theorem 18 at the end of the paper.

5. THEOREM. If $\pi$ is the field projection for a field of topological spaces $(E, B, Y, \Phi)$, then $(E, B, \pi)$ is a field of spaces in the sense of Dauns and Hofmann.

Proof. It is suffices to show that there is an adequate supply of 
local sections. For $x \in E$, select $\phi \in \Phi$ so that $x \in$ image $\phi$ and fix $y \in$ $Y$ so that $(b, y) \in \phi^{-1}(x)$ for some $b \in B$. Define $\sigma: U \rightarrow E$ on $U=$ $\{b: b \in B,(b, y) \in \operatorname{dom} \phi\}$ by $\sigma(b)=\phi(b, y)$. Then $\sigma$ is a continuous local section which contains $x$ in its image. Therefore axiom (I) is satisfied.

Next we deal with the problem of assigning topologies to $E, B$, and $Y$ for a given field of sets $(E, B, Y, \Phi)$ so that the latter becomes a field of topological spaces. First we note that the topology on $E$ is entirely determined by $\Phi$ and the topologies on $B$ and $Y$.

6. THEOREM. Let $B$ and $Y$ be topological spaces and $(E, B, Y$, $\Phi)$ be a field of sets. Then the topology on $E$ is uniquely determined in the sense that if $\mathscr{T}_{1}$ and $\mathscr{T}_{2}$ are topologies on $E$ for which $(E, B$, $Y, \Phi)$ is a field of topological spaces, then the two topologies are identical.

Proof. Due to symmetry it suffices to show that $\mathscr{G}_{1}$ is weaker than $\mathscr{T}_{2}$. Let $U \in \mathscr{F}_{1}$ and $x \in U$. Select $\phi \in \Phi$ such that $x \in$ image $\phi$. Since $\phi$ is continuous with respect to $\mathscr{S}_{1}$ and open with respect to $\mathscr{T}_{2}$, it follows that $\phi \phi^{-1}(U) \in \mathscr{S}_{2}$. Since $x \in \phi \phi^{-1}(U) \subset U$, the set $U$ is a $\mathscr{T}_{2}$-neighborhood of each of its points. Whence $U \in \mathscr{F}_{2}$.

Thus, in constricting a field of spaces, it is enough to know that $(E, B, Y, \Phi)$ is a field of sets and that $B$ and $Y$ are topological spaces such that each $\phi \in \Phi$ generates a lower semicontinuous decomposition of its domain. For if this is the case, $(E, B, Y, \Phi)$ becomes a field of spaces if $E$ is assigned the final topology (i.e., the finest topology such that all $\phi \in \Phi$ are continuous) and Theorem 6 asserts that the final topology is the only admissible topology on $E$.

We now give a series of technical lemmas that are needed to determine which topologies on $B$ and $Y$ give rise to a field of spaces $(E, B, Y, \Phi)$.

7. Lemma. If $(E, B, Y, \Phi)$ is a field of sets and $V$ is a subset of $B$, then $\pi^{-1}(V)=\bigcup_{\phi \in \phi} \phi(V \times Y)$.

Proof. Since $\pi \circ \phi$ is a restriction of the projection of $B \times Y$ on $B$, it is immediate that $\pi^{-1}(V) \supset \bigcup \phi(V \times Y)$. If $x \in \pi^{-1}(V)$, then (A) implies that there exists $\phi \in \Phi$ such that $x \varepsilon$ image $\phi$. If $(b, y) \in \dot{\phi}^{-1}(x)$ then $b=\pi \circ \phi(b, y)=\pi(x) \in V$. Thus $x \in \dot{\phi}(V \times Y)$.

8. Lemma. If $(E, B, Y, \Phi)$ is a field of sets, $V \subset B$ and $W \subset Y$ then for any $\dot{\phi}_{i} \in \Phi, i=1,2$, it follows that

$$
\phi_{2}^{-1} \dot{\phi}_{1}(V \times W)=(V \times Y) \cap \phi_{2}^{-1} \phi_{1}(B \times W) .
$$


Proof. If $(b, y)$ belongs to the set on the left, then clearly $(b, y) \in$ $\phi_{2}^{-1} \phi_{1}(B \times W)$. Also $\pi \circ \phi_{2}(b, y)=\pi \circ \phi_{1}(b, w) \in V$ for some $w \in W$. Thus $(b, y)$ belongs to the set on the right. Conversely, if $(b, y)$ belongs to the set on the right, then $b \in V$ and $\phi_{2}(b, y)=\phi_{1}(b, w)$ for some $w \in W$. Thus $(b, y) \in \phi_{2}^{-1} \phi_{1}(V \times W)$.

9. Corollary. (a) $\phi_{1}(B \times W) \cap \phi_{2}(V \times Y)=\phi_{1}(V \times W) \cap$ image $\phi_{2}$ and (b) $\phi(V \times W)=\phi(B \times W) \cap \phi(V \times Y)=\phi(B \times W) \cap \pi^{-1}(V)$ for all $V \subset B$, all $W \subset Y$ and for $\phi, \phi_{1}, \phi_{2}$ in $\Phi$.

10. Theorem. Let $(E, B, Y, \Phi)$ be a field of sets where $B$ and $Y$ are topological spaces. $A$ necessary and sufficient condition that $E$ admit a topology with respect to which $(E, B, Y, \Phi)$ is a field of spaces is that $\phi_{2}^{-1} \phi_{1}(B \times W)$ be open in $B \times Y$ for each pair $\phi_{1}, \phi_{2}$ in $\Phi$ and each open subset $W$ of $Y$.

Proof. Necessity. Since $\phi_{1}$ is an open map and $\phi_{2}$ is continuous, the set $\phi_{2}^{-1} \phi_{1}(B \times W)$ must be open.

Sufficiency. Let the topology on $E$ be the one which has $\mathscr{S}=$ $\{\phi(G): \phi \in \Phi, G$ open in $B \times Y\}$ as a subbase. With respect to this topology all $\phi \in \Phi$ are open maps. Also, dom $\phi=\phi^{-1} \phi(B \times Y)$ is open for each $\phi \in \Phi$. Whence it suffices to show that each $\phi \in \Phi$ is continuous. For $\phi \in \Phi$ and $(b, y) \in \operatorname{dom} \phi$, let $S$ be a neighborhood of $\phi(b, y)$ which belongs to the subbase $\mathscr{S}$. Then there exists $\phi_{1} \in \Phi$ and $G$ open in $B \times Y$ such that $S=\phi_{1}(G)$. Thus there is a point $\left(b_{1}, y_{1}\right) \in G$ such that $\phi_{1}\left(b_{1}, y_{1}\right)=\phi(b, y)$. Now axiom (C) implies $b=b_{1}$. Since $G$ is open in the product space, there are open sets $V$ and $W$ in $B$ and $Y$ respectively such that $\left(b, y_{1}\right) \in V \times W \subset G$. Thus by Lemma $8,(b, y) \in$ $\dot{\phi}^{-1} \dot{\phi}_{1}(V \times W)=V \times Y \cap \dot{\phi}^{-1} \dot{\phi}_{1}(B \times W)$. By the hypothesis, the latter set is open. Hence $\phi^{-1} \phi_{1}(V \times W)$ is a neighborhood of $(b, y)$ whose image under $\phi$ (namely $\phi_{1}(V \times W) \cap$ image $\phi$ ) is contained in $S=\phi_{1}(G)$. This proves that each $\phi \in \Phi$ is continuous.

11. Theorem. If $(E, B, Y, \Phi)$ is a field of topological spaces and $B_{1}$ is a space with underlying set $B$, whose topology is finer than that on $B$, then $\left(E_{1}, B_{1}, Y, \Phi\right)$ is also a field of topological spaces when $E_{1}$ is the set $E$ with the final topology with respect to $\Phi$ and $B_{1} \times Y$. Moreover, $\left\{U \cap \pi^{-1}(V): U\right.$ is open in $E, V$ is open in $\left.B_{1}\right\}$ is a base for the topology on $E_{1}$.

Proof. The first part of the theorem follows from Lemma 10. For if $W$ is open in $Y$, then $\phi_{2}^{-1} \phi_{1}(B \times W)$ is open in $B \times Y$; hence this set is also open in $B_{1} \times Y$. By Lemma $3, \pi$ is a continuous map 
from $E_{1}$ to $B_{1}$. Thus each $U \cap \pi^{-1}(V)$ is open in $E_{1}$ if $U$ is open in $E$ and $V$ is open in $B_{1}$. To see that these sets form a base, let $G$ be open in $E_{1}$ and $x \in G$. Then there exist elements $b \in B, y \in Y$, and $\phi \in \Phi$ such that $\phi(b, y)=x$. Let $V$ and $W$ be open $B_{1}$ and $Y$ respectively such that $(b, y) \in V \times W \subset \phi^{-1}(G)$. Then by Corollary 9, we have $x \in$ $\phi(B \times W) \cap \pi^{-1}(V)=\phi(V \times W) \subset G$. Since $\phi(B \times W)$ is open in $E$, the proof is complete.

12. Lemma. Let $(E, B, Y, \Phi)$ be a field of sets. For a given topology on $Y$, a necessary and sufficient condition that $E$ and $B$ admit topologies for which $(E, B, Y, \Phi)$ is a field of spaces is that $p\left(\phi_{2}^{-1} \dot{\phi}_{1}(\{b\} \times W)\right)$ be open in $Y$ (here $p$ denotes the projection of $B \times$ $Y$ on $Y$ ) for each $b \in B$, each open set $W$ in $Y$ and each pair $\phi_{1}, \phi_{2}$ in $\Phi$.

Proof. Necessity. Note that $p\left(\phi_{2}^{-1} \phi_{1}(\{b\} \times W)\right)=\left\{y:(b, y) \in \phi_{2}^{-1}\right.$ $\left.\phi_{1}(B \times W)\right\}$. The latter set is open since $\phi_{1}$ an is open map and $\phi_{2}$ is continuous.

Sufficiency. If $B$ is assigned the discrete topology then $\phi_{2}^{-1} \phi_{1}(B \times$ $W)=\bigcup_{b \in B}\{b\} \times p\left(\phi_{2}^{-1} \phi_{1}(\{b\} \times W)\right)$ is open in $B \times Y$. According to Lemma 10 , this is sufficient to insure that $E$ admits a topology for which $(E, B, Y, \Phi)$ is a field of spaces.

13. Definition. Let $(E, B, Y, \Phi)$ be a field of sets. A topology on $Y$ is called $\Phi$-admissible if satisfies the condition stated in Lemma 12. For a given $\Phi$-admissible topology on $Y$, the coarsest topology (if such exists) on $B$ for which $(E, B, Y, \Phi)$ becomes a field of topological spaces is called the weak topology on $B$ determined by $\Phi$.

The term "weak topology" is motivated by Theorem 11 which says, in effect, that if the weak topology on $B$ exists, then any finer topology on $B$ gives rise to a field of topological spaces.

14. Theorem. Let $(E, B, Y, \Phi)$ be a field of sets. If the topology on $Y$ is locally compact and $\Phi$-admissible, then the weak topology on $B$ has a subbase $\mathscr{S}$ consisting of all sets of the form $V\left(K, W, \phi_{1}, \phi_{2}\right)=$ $\left\{b:\{b\} \times K \subset \phi_{2}^{-1} \phi_{1}(B \times W)\right\}$ where $K$ is compact, $W$ is open in $Y$ and $\phi_{1}, \phi_{2}$ are in $\Phi$ (i.e., the weak topology is the same as the "compactopen" topology).

Proof. Let $\mathscr{T}$ be a topology on $B$ for which $(E, B, Y, \Phi)$ is a field of spaces (with respect to the final topology on $E$ ). If $b \in V=$ $V\left(K, W, \phi_{1}, \phi_{2}\right)$ then $\{b\} \times K$ is contained in the open set $\phi_{2}^{-1} \phi_{1}(B \times W)$ in $B \times Y$. By Wallace's theorem ([5], p. 142) it follows that $V$ is open in $\mathscr{T}$. Thus the topology generated by $\mathscr{S}$ is weaker than $\mathscr{T}$. It remains to show that the topology generated by $\mathscr{S}$ is compatible 
with the field structure. Let $W$ be open in $Y$ and $\phi_{1}, \phi_{2}$ be elements of $\Phi$. We must show that $\phi_{2}^{-1} \phi_{1}(B \times W)$ is open; let $(b, y)$ be an element of this set. Since the topology on $Y$ is $\Phi$-admissible, Lemma 12 implies there exists an open set $W_{1}$ in $Y$ containing $y$ such that $\{b\} \times W_{1} \subset \phi_{2}^{-1} \phi_{1}(B \times W)$. Since $Y$ is locally compact, there is a compact neighborhood $K$ of $y$ contained in $W_{1}$. Then for $V=V\left(K, W, \phi_{1}, \phi_{2}\right)$, the set $V \times K$ is a neighborhood of $(b, y)$ contained in $\phi_{2}^{-1} \phi_{1}(B \times W)$. Whence the latter set is open. It now follows from Lemma 10 that $\mathscr{S}$ is a subbase for the weak topology.

In case that $Y$ has an algebraic structure as well as a topological one, it is sometimes possible to characterize the weak topology even when $Y$ is not locally compact. Specifically let $Y$ be a commutative topological group (written additively) and $B$ be an index set for a family of subgroups $\left\{G_{b}: b \in B\right\}$ and define $E=\bigcup_{b \in B}\{b\} \times\left(Y / G_{b}\right)$ and $\phi: B \times Y \rightarrow E$ by $\phi(b, y)=\left(b, y+G_{b}\right)$. If the stalks $\{b\} \times Y / G_{b}$ are assigned the natural quotient group structures then $\phi(b, \cdot)$ is a homomorphism for each $b \in B$.

15. THEOREM. If $\left\{G_{b}: b \in B\right\}$ is a family of closed subgroups of the commutative topological group $Y$, then the quadruple $(E, B, Y, \phi)$ defined above is a field of sets for which the topology on $Y$ is $\phi$ admissible and the weak topology on $B$ has the set $\mathscr{S}$ described in Theorem 14 as a subbase.

Proof. Since dom $\phi=B \times Y$ and image $\phi=E$, axioms (A) and (B) for a field of sets are satisfied. It is clear that axiom (C) holds. Also, since translation is continuous in $Y$, it follows from Lemma 12 that the topology on $Y$ is $\phi$-admissible. As in Theorem 14, it can be verified that the topology generated by $\mathscr{S}$ is weaker than any topology which is compatible with the field structure. Thus it suffices to show that $\phi$ is open and continuous with respect to the topology generated by $\mathscr{S}$. Let $W$ be open in $Y$ and let $(b, y) \in \phi^{-1} \phi(B \times W)$. Then there exists $w \in W$ such that $\phi(b, y)=\phi(b, w)$. Let $N$ be a neighborhood of 0 in $Y$ such that $w+N+N \subset W$. Set $V=V(y, w+N, \phi, \phi)$ (in the notation of Theorem 13). If $\left(b^{\prime}, y^{\prime}\right) \in V \times(y+N)$, then $y \in w+N+$ $G_{b^{\prime}}$ since $b^{\prime} \in V$, and $y^{\prime} \in y+N$. Thus $y^{\prime} \in w+N+N+G_{b^{\prime}} \subset W+G_{b^{\prime}}$. Whence $\phi\left(b^{\prime}, y^{\prime}\right) \in \phi(B \times W)$. This proves that $V \times(y+N) \subset \phi^{-1} \phi(B \times$ $W)$. The theorem now follows from Lemma 10 .

In the remaining part of this paper we shall explore the connection between our definition of a field of topological spaces and the DaunsHofmann definition of field of uniform spaces. To facilitate this discussion we shall reproduce some of the definitions in the DaunsHofmann memoir [3]. 
16. Definition. (a) Let $E, B$ be topological spaces and $\pi: E \rightarrow$ $B$ be an open continuous surjection. A set of global section $\Sigma$ is a full set if $E=\bigcup_{\sigma \in \Sigma} \sigma(B)$. Then $(E, B, \pi)$ is a field with a full set of sections.

(b) Let $\mathscr{U}$ be a uniformity (in the sense of Weil, page 169 of [1]) on $E$. Then $\mathscr{C}$ is a field uniformity if $E \vee E \in \mathscr{C}$ where $E \vee$ $E=\{(x, y):(x, y) \in E \times E$ such that $\pi(x)=\pi(y)\}$.

(c) If $(E, B, \pi)$ is a field with a full set $\Sigma$ of continuous sections and $\mathscr{U}$ is a field uniformity on $E$ which satisfies the technical condition stated in Lemma 1.9 of [3], then $(E, \pi, B, \mathscr{C})$ is called a field of uniform spaces.

(d) If $\Sigma$ a full set of continuous section for a field $(E, \pi, B, \mathscr{C})$ and $U \in \mathscr{U}, \sigma \in \Sigma$ set $\widetilde{U}(\sigma)=\{\tau: \tau \in \Sigma,(\sigma(b), \tau(b)) \in U$ for all $b \in B\}$. Then the topology on $\Sigma$ is the one for which $\{\widetilde{U}(\sigma): U \in \mathscr{Z}\}$ is a neighborhood base at $\sigma$.

(e) Let $\Sigma, E, \pi, B$, and $\mathscr{C}$ be as in (d). For $U \in \mathscr{Q}, \sigma \in \Sigma$ define $U(\sigma)=\{x: x \in E,(\sigma(b), x) \in U$ for some $b \in B\}$.

Next we shall form a field of topological spaces from a given field of uniform spaces. Let $(E, \pi, B, \mathscr{Z})$ be a field of uniform spaces with a full set of global sections $\Sigma$ and define $\phi: B \times \Sigma \rightarrow E$ by $\phi(b, \sigma)=\sigma(b)$.

17. Lemma. If $U^{\circ}$ denotes the quasi-interior of $U$ as defined in Dauns-Hofmann ([3], p. 19) then $U^{\circ}(\sigma) \subset \phi(B \times \widetilde{U}(\sigma)) \subset U(\sigma)$ for each $\sigma \in \Sigma$ and each $U \in \mathscr{W}$.

Proof. First assume $y \in U^{\circ}(\sigma)$ and set $x=\sigma(\pi(y))$. Then $(x, y) \in$ $U^{\circ}$. Thus by Definition 2.5, p. 19 in [3], there exists $V \in \mathscr{Q} C$ and $\tau \in \Sigma$ such that $y=\tau(\pi(y))$ and $V(\tau) \subset U(\sigma)$. Now $\tau[B] \subset V(\tau) \subset U(\sigma)$ implies $\tau \in \widetilde{U}(\sigma)$. Whence $y=\phi(\pi(y), \tau) \in \phi(B \times \widetilde{U}(\sigma))$. This proves the first of the two inclusions. Next assume $y \in \dot{\phi}(B \times \widetilde{U}(\sigma))$ and let $(b, \tau) \in B \times \widetilde{U}(\sigma)$ be such that $y=\phi(b, \tau)=\tau(b)$. Now $\tau \in \widetilde{U}(\sigma)$ implies that $(\sigma(b), y)=(\sigma(b), \tau(b)) \in U$. Whence $y \in U(\sigma)$.

18. TheOREM. If $(E, \pi, B, \mathscr{Q})$ is a field of uniform spaces with a full set $\Sigma$ of continuous global sections, for which $\mathscr{C}_{0}=\{U$ : $U \in \mathscr{U}$ such that $\left.U^{\circ} \in \mathscr{U}\right\}$ is a base for $\mathscr{U}$, then $(E, B, \Sigma, \phi)$ is a field of topological spaces.

Proof. It is easy to verify that $(E, B, \Sigma, \phi)$ is a field of sets for which dom $\phi=B \times \Sigma$. Thus it suffices to show that $\phi$ is continuous and open. For $(b, \sigma) \in B \times \Sigma$ and a neighborhood $N$ of $\dot{\phi}(b, \sigma)$ in $E$, then by Lemma 1.9, page 4 in [3], there exists a neighborhood $V$ of $b$ and $U \in \mathscr{U}$ such that $\pi^{-1}(V) \cap U(\sigma) \subset N$. Now $V \times \widetilde{U}(\sigma)$ is a neighborhood of $(b, \sigma)$ in $B \times \Sigma$ whose image satisfies $\phi(V \times \widetilde{U}(\sigma))=\pi^{-1}(V) \cap \phi(B \times$ 
$\widetilde{U}(\sigma)) \subset \pi^{-1}(V) \cap U(\sigma)$ by Corollary 9 and Lemma 17; whence $\phi$ is continuous. Since $\phi(B \times\{\sigma\}) \subset U^{\circ}(\sigma)$, it follows from Lemma 17 and the fact $\mathscr{U}_{0}$ is a base that $\phi(B \times W)$ is open in $E$ for every open set $W$ in $\Sigma$. Thus by Corollary $9(\mathrm{~b}), \phi$ is an open map. Whence $(E, B$, $\Sigma, \phi)$ is a field of topological spaces.

There are several conditions on the quasi-interiors $U^{\circ}$ which are equivalent to the assumption that $\mathscr{U}_{0}$ is a base for $\mathscr{C}$. The following lemma is contained implicitly in the material on pages 19 and 20 of [3].

19. Lemma. The following conditions are equivalent:

(a) $\left\{U: U \in \mathscr{Q}, U^{\circ} \in \mathscr{Q}\right\}$ is a base for $\mathscr{U}$.

(b) $\left\{U^{\circ}: U \in \mathscr{Q}\right\}$ is a base for $\mathscr{U}$

(c) $U^{\circ} \in \mathscr{U}$ for all $U \in \mathscr{U}$.

It should be noted that the requirement in the Dauns-Hofmann development that the set of quasi-interiors $\mathscr{U}_{0}$ form a base for $\mathscr{C}$ is precisely the condition that insures that the map $\phi$ is an open map.

This shows that under any of the equivalent conditions of Lemma 19 that each field of uniform spaces (in the sense of Dauns and Hofmann) is a field of topological spaces. The converse is true in the following setting: If $(E, B, Y, \phi)$ is a field of topological spaces with $Y$ a commutative topological group and $\phi(b, \cdot)$ is a homomorphism for each $b \in B$ then there is a natural field uniformity $\mathscr{C}$ on $E$ such that $(E, \pi, B, \mathscr{C})$ is a field of uniform spaces.

Specifically, let $Y$ be a commutative topological group written additively and let $(E, B, Y, \phi)$ be a field of topological spaces such that $\operatorname{dom} \phi=B \times Y$ and $\phi(b, \cdot)$ is a homomorphism with kernel $G_{b}$ for each $b \in B$. Further, let $\mathscr{N}$ be a base for the neighborhoods of 0 in $Y$. For $N \in \mathscr{N}$ define $U_{N}$ to be the subset of $E \times E$ given by $\left\{\left(x_{1}, x_{2}\right)\right.$ : there exists $y_{1}, y_{2}$ in $Y, b \in B$ such that $y_{1}-y_{2} \in N$ and $x_{i}=$ $\phi\left(b, y_{i}\right)$ for $\left.i=1,2\right\}$. It is easy to verify that $\left\{U_{N}: N \in \mathscr{N}\right\}$ is a base for a field uniformity $\mathscr{U}$ on $E$. For $y \in Y$, define $\hat{y}(b)=\phi(b, y)$. Then clearly $\hat{Y}=\{\hat{y}: y \in Y\}$ is a full set of global continuous sections. If $\bigcap_{b \in B} G_{b}=\{0\}$, then $y \rightarrow \hat{y}$ is bijection between $Y$ and $\hat{Y}$.

20. Lemma. If ( $E, B, Y, \phi)$ is a field of topological spaces, $Y$ is a topological group and $\mathscr{Q}$ is defined as in the preceding paragraph, then $(E, \pi, B, \mathscr{Q})$ is a field of uniform spaces (in the sense of Dauns and Hofmann) for which $Y$ is a full set of global sections.

Proof. It suffices to show that the condition stated in Lemma 1.9 of [3] is satisfied. Specifically, we must show that the sets $U_{N}(\hat{y}) \cap$ $\pi^{-1}(V)$ form a base for the topology on $E$. Now $U_{N}(\hat{y})$ equals, by definition, $\phi(B \times[y+N])$. Thus by Corollary 9 (cf. proof of Theorem 
11), the sets $U_{N}(\hat{y}) \cap \pi^{-1}(V)$ form a base for the topology on $E$.

21. Lemma. If $(E, B, Y, \phi)$ is a field of topological spaces satisfying the hypothesis of Lemma 19 , then $\widetilde{U}_{N}(\hat{y})=\left(y+\bigcap_{b \in B}\left[N+G_{b}\right]\right)^{\wedge}$.

Proof. By definition $\hat{z} \in \widetilde{U}_{N}(\hat{y})$ if and only for each $b \in B$ there are elements $y_{b}, z_{b}$ of $Y$ such that $z_{b}-y_{b} \in N$ while $\phi(b, y)=\phi\left(b, y_{b}\right)$ and $\phi(b, z)=\phi\left(b, z_{b}\right)$. Thus $z-y=z_{b}-y_{b}+\left(z-z_{b}\right)+\left(y_{b}-y\right) \in N+G_{b}$. Thus $\widehat{z} \in \widetilde{U}_{N}(\hat{y})$ if and only if $z-y \in \bigcap_{b \in B}\left[N+G_{b}\right]$. This completes the proof.

The next theorem summarizes the results contained in the discussion preceding Lemma 20. In the setting under consideration, this theorem says that fields of topological spaces (as defined in this paper) are equivalent to the fields of uniform spaces defined by Dauns and Hofmann in [3].

22. THEorem. Let $(E, B, Y, \phi)$ be a field of topological spaces with $Y$ a (Hausdorff) commutative topological group and $\phi$ be a map from $B \times Y$ to $E$ such that $\phi(b, \cdot)$ is a homomorphism with kernel $G_{b}$ for each $b \in B$. If $\mathscr{N}$ is a base for the neighborhoods of 0 in $Y$ such that $N=\bigcap_{b \in B}\left[N+G_{b}\right]$ for each $N \in \mathscr{N}$ and $\mathscr{Q}$ is the field uniformity which has $\left\{U_{N}: N \in \mathscr{N}\right\}$ as a base (for definition of $U_{N}$ see the paragraph preceding Lemma 20$)$, then $(E, \pi, B, \mathscr{Q})$ is a field of uniform spaces for which $\hat{Y}$ is a full set of global sections. Moreover, the $\tilde{\mathscr{C}}$ uniform topology is the same as the original topology on $Y$.

Proof. Except for the last assertion, this theorem follows directly from Lemma 20. Since $N$ is the intersection of all the sets $N+G_{b}$, we have by Lemma 21 that $\widetilde{U}(\widehat{y})=(y+N)^{\wedge}$. It is now clear that the uniform topology on $Y$ and the original topology are identical.

\section{REFERENCES}

1. N. Bourbaki, General Topology, Part 1, Hermann, Paris, 1966

2. Glen E. Bredon, Sheaf Theory, McGraw-Hill, New York, 1967.

3. J. Dauns and K. H. Hofmann, Representation of rings by sections, Memoirs Amer. Math. Soc., Number 83, 1968.

4. C. H. Dowker, Lectures on Sheaf Theory, Tata Institute Lecture Notes, Bombay, 1956.

5. J. L. Kelley, General Topology, Von Nostrand, New York, 1955.

6. J. L. Koszul, Lectures on Fibre Bundles and Differential Geometry, Tata Institute Lecture Notes, Bombay, 1960.

7. N. Steenrod, The Topology of Fibre Bundles, Princeton, 1951.

8. Richard G. Swan, The Theory of Sheaves, The University of Chicago Press, 1964.

Received February 8, 1972 and in revised form August 1, 1972. This research was supported in part by NSF Grant GP-19549. 


\section{PACIFIC JOURNAL OF MATHEMATICS}

\section{EDITORS}

D. Gilbarg and J. Milgram

Stanford University

Stanford, California 94305

\author{
R. A. Beaumont \\ University of Washington \\ Seattle, Washington 98105
}

J. DuGUNDJI

Department of Mathematics

University of Southern California

Los Angeles, California 90007

Richard ARENS

University of California

Los Angeles, California 90024

\section{ASSOCIATE EDITORS}
E. F. BECKENBACH
B. H. NeumanN
F. WOLF
K. YosHIDA

\section{SUPPORTING INSTITUTIONS}

UNIVERSITY OF BRITISH COLUMBIA

CALIFORNIA INSTITUTE OF TECHNOLOGY

UNIVERSITY OF CALIFORNIA

MONTANA STATE UNIVERSITY

UNIVERSITY OF NEVADA

NEW MEXICO STATE UNIVERSITY

OREGON STATE UNIVERSITY

UNIVERSITY OF OREGON

OSAKA UNIVERSITY

\author{
UNIVERSITY OF SOUTHERN CALIFORNIA \\ STANFORD UNIVERSITY \\ UNIVERSITY OF TOKYO \\ UNIVERSITY OF UTAH \\ WASHINGTON STATE UNIVERSITY \\ UNIVERSITY OF WASHINGTON
* * * *
AMERICAN MATHEMATICAL SOCIETY \\ NAVAL WEAPONS CENTER
}

The Supporting Institutions listed above contribute to the cost of publication of this Journal, but they are not owners or publishers and have no responsibility for its content or policies.

Mathematical papers intended for publication in the Pacific Journal of Mathematics should be in typed form or offset-reproduced, (not dittoed), double spaced with large margins. Underline Greek letters in red, German in green, and script in blue. The first paragraph or two must be capable of being used separately as a synopsis of the entire paper. The editorial "we" must not be used in the synopsis, and items of the bibliography should not be cited there unless absolutely necessary, in which case they must be identified by author and Journal, rather than by item number. Manuscripts, in duplicate if possible, may be sent to any one of the four editors. Please classify according to the scheme of Math. Rev. Index to Vol. 39. All other communications to the editors should be addressed to the managing editor, Richard Arens, University of California, Los Angeles, California, 90024.

50 reprints are provided free for each article; additional copies may be obtained at cost in multiples of 50 .

The Pacific Journal of Mathematics is issued monthly as of January 1966. Regular subscription rate: $\$ 48.00$ a year (6 Vols., 12 issues). Special rate: $\$ 24.00$ a year to individual members of supporting institutions.

Subscriptions, orders for back numbers, and changes of address should be sent to Pacific Journal of Mathematics, 103 Highland Boulevard, Berkeley, California, 94708.

PUBLISHED BY PACIFIC JOURNAL OF MATHEMATICS, A NON-PROFIT CORPORATION

Printed at Kokusai Bunken Insatsusha (International Academic Printing Co., Ltd.), 270, 3-chome Totsuka-cho, Shinjuku-ku, Tokyo 160, Japan. 


\section{Pacific Journal of Mathematics}

\section{Vol. 46, No. 2 December, 1973}

Christopher Allday, Rational Whitehead products and a spectral sequence of

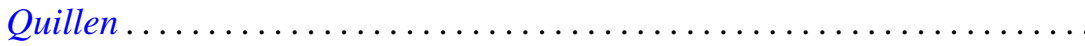

James Edward Arnold, Jr., Attaching Hurewicz fibrations with fiber

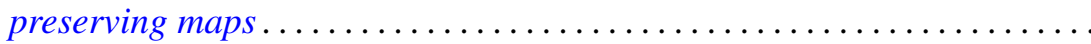

Catherine Bandle and Moshe Marcus, Radial averaging transformations with various metrics.................................

David Wilmot Barnette, A proof of the lower bound conjecture for convex

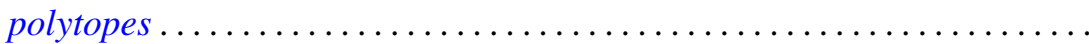

Louis Harvey Blake, Simple extensions of measures and the preservation of

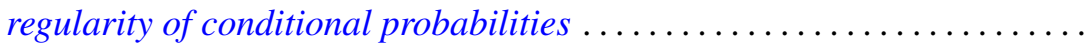

James W. Cannon, New proofs of Bing's approximation theorems for

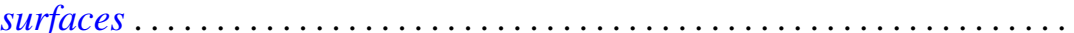

C. D. Feustel and Robert John Gregorac, On realizing HNN groups in

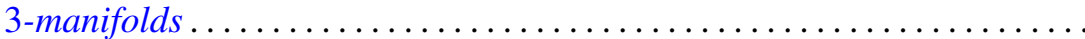

Theodore William Gamelin, Iversen's theorem and fiber algebras . . . . . . . . 389

Daniel H. Gottlieb, The total space of universal fibrations . . . . . . . . . . . .

Yoshimitsu Hasegawa, Integrability theorems for power series expansions of

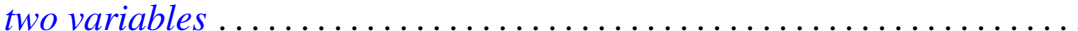

Dean Robert Hickerson, Length of period simple continued fraction expansion of $\sqrt{ } d$

Herbert Meyer Kamowitz, The spectra of endomorphisms of the disc algebra.

Dong S. Kim, Boundedly holomorphic convex domains

Daniel Ralph Lewis, Integral operators on $\mathscr{L}_{p}$-spaces ...

John Eldon Mack, Fields of topological spaces . . . . . . . . .

V. B. Moscatelli, On a problem of completion in bornology

Ellen Elizabeth Reed, Proximity convergence structures. .

Ronald C. Rosier, Dual spaces of certain vector sequence spaces .

Robert A. Rubin, Absolutely torsion-free rings

Leo Sario and Cecilia Wang, Radial quasiharmonic functions . .

James Henry Schmerl, Peano models with many generic classes .

H. J. Schmidt, The $\mathscr{F}$-depth of an $\mathscr{F}$-projector ............

Edward Silverman, Strong quasi-convexity. . . . . . . . . . . . . . . . . 549

Barry Simon, Uniform crossnorms ....................... 555

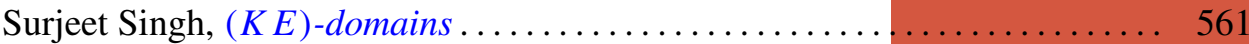

Ted Joe Suffridge, Starlike and convex maps in Banach spaces . . . . . . . . 575

Milton Don Ulmer, $C$-embedded $\Sigma$-spaces . . . . . . . . . . . . . . . . 591

Wolmer Vasconcelos, Conductor, projectivity and injectivity . . . . . . . . . 603 